\title{
Thiol-Based Peroxidases and Ascorbate Peroxidases: Why Plants Rely on Multiple Peroxidase Systems in the Photosynthesizing Chloroplast?
}

\author{
Karl-Josef Dietz*
}

\begin{abstract}
Photosynthesis is a highly robust process allowing for rapid adjustment to changing environmental conditions. The efficient acclimation depends on balanced redox metabolism and control of reactive oxygen species release which triggers signaling cascades and potentially detrimental oxidation reactions. Thiol peroxidases of the peroxiredoxin and glutathione peroxidase type, and ascorbate peroxidases are the main peroxide detoxifying enzymes of the chloroplast. They use different electron donors and are linked to distinct redox networks. In addition, the peroxiredoxins serve functions in redox regulation and retrograde signaling. The complexity of plastid peroxidases is discussed in context of suborganellar localization, substrate preference, metabolic coupling, protein abundance, activity regulation, interactions, signaling functions, and the conditional requirement for high antioxidant capacity. Thus the review provides an opinion on the advantage of linking detoxification of peroxides to different enzymatic systems and implementing mechanisms for their inactivation to enforce signal propagation within and from the chloroplast.
\end{abstract}

\section{INTRODUCTION}

Photosynthesis depends on dynamic input parameters. In particular photon flux density may change in fractions of seconds. Many other parameters like $\mathrm{CO}_{2}$-availability at the site of carbon fixation, temperature, local effects of pathogens and nutrient supply can fluctuate on the time scale of minutes to days and strongly affect photosynthesis. Photosynthesis is most efficiently regulated to avoid damage despite these variations (Allahverdiyeva et al., 2015). The robustness is realized by

Biochemistry and Physiology of Plants, Faculty of Biology, W5-134, Bielefeld University, University Street 25, 33501 Bielefeld, Germany

*Correspondence: karl-josef.dietz@uni-bielefeld.de

Received 27 November, 2015; accepted 23 December, 2015; published online 25 January, 2016

Keywords: acorbate peroxidase, chloroplast, peroxiredoxin, photosynthesis, redox sensing regulatory networks which exploit information from systems performance and adjust activities of critical processes. Reactive oxygen species and redox regulation by thiol switches play a major role in the control of photosynthesis (Dietz and Hell, 2015). In this context, the question arises as to why chloroplasts rely on two types of peroxidases, the thiol-based and ascorbate-dependent peroxidases.

\section{SUPEROXIDE AND HYDROGEN PEROXIDE GENERATION IN PHOTOSYNTHESIS}

The photosynthetic electron transport chain has two main mechanisms to produce superoxide anions. (i) Reduced plastoquinone donates electrons to $\mathrm{O}_{2}$ via the plastid terminal oxidase (PTOX) (Heyno et al., 2009). Thus the regulatory circuitry controlling the redox state of the intersystem electron transport carrier plastoquinone tends to adjust an intermediately reduced level of reduced to oxidized plastoquinone to minimize ROS generation. (ii) (Over-)reduced photosystem I (PSI) as consequence of an imbalance between light driven electron delivery and electron acceptor availability such as $\mathrm{NADP}^{+}$, nitrite or sulfite transfers electrons from ferredoxin or the Fe-S-center Fx to $\mathrm{O}_{2}$. This $\mathrm{O}_{2}$-reduction reaction is called the Mehler reaction. The magnitude of the Mehler reaction is discussed in a controversial manner ranging from rather low rates (Laisk et al., 2006) to high rates reaching $30 \%$ of total electron flow (Badger et al., 2000). A comparison of 101 species for their capacity of $\mathrm{O}_{2-}$ dependent electron drainage from the photosynthetic electron transport revealed species-specific differences, e.g. between gymnosperms and angiosperms (Shirao et al., 2013). While $\mathrm{O}_{2-}$ dependent electron flow ranged around $3 \%$ in diverse gymnosperms, the rates dropped to about $1 \%$ or less in angiosperms such as tobacco (Shirao et al., 2013). These authors also elaborated on the question whether PSI- and PTOX-dependent $\mathrm{O}_{2}$-reduction can experimentally be distinguished in wild type plants. Based on the similar affinity to $\mathrm{O}_{2}$ they argued that only the combined $\mathrm{O}_{2}$-reduction can be measured. The rate of the Mehler reaction changes in dependence on environmental conditions such as light, temperature, nutritional status, and restrictions in $\mathrm{CO}_{2}$-supply, e.g. following stress-induced stomatal closure. Surprisingly, Ruuska et al. (2000) observed no change in light-dependent $\mathrm{O}_{2}$ uptake in transgenic $A$. thaliana 

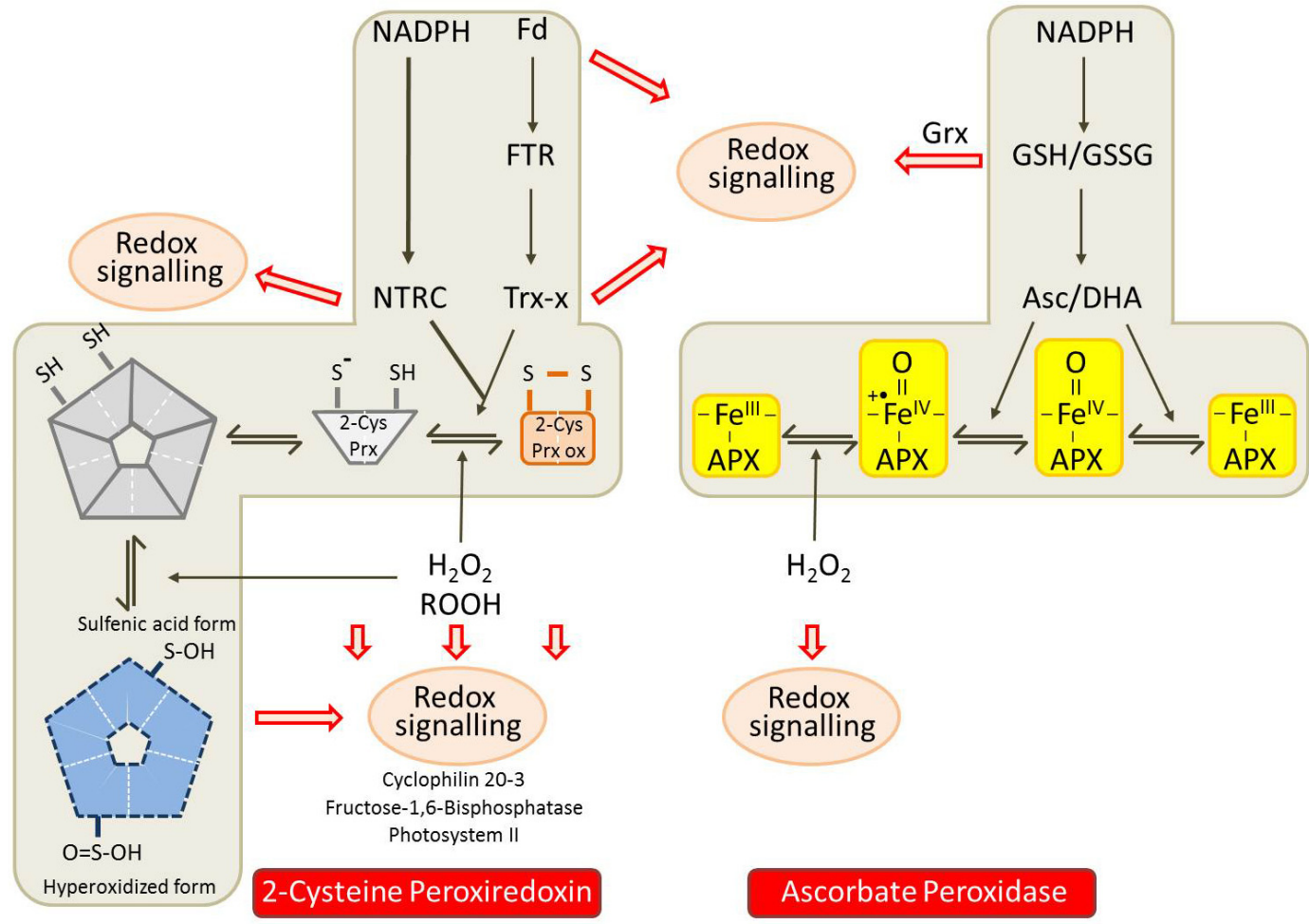

Fig. 1. Inactivation mechanisms for chloroplast ascorbate peroxidase and 2-cysteine peroxiredoxin. The scheme depicts the catalytic and conformational cycle of 2-CysPrx (left hand side) and the transition state of ascorbate peroxiodase on the right hand side. The 2-CysPrx adopts the dimeric disulfide bonded form $\left(\mathrm{Prx}_{\mathrm{ox}}\right)$, the reduced active form as dimer or decamer or the hyperoxidized form (Muthuramalingam et al. 2009). The Fe in the catalytic center of APX converts to the oxidized radical ${ }^{\circ+} \mathrm{Fe}^{\mathrm{IV}}$ which is prone to inactivation if ascorbate is missing. Also indicated are the coupled reactions and the signaling functions in the cell. See text for details. Asc, ascorbate; DHA, dehydroascorbate; Fd, ferredoxin; FTR, ferredoxin-dependent thioredoxin reductase; Grx, glutaredoxin; NTRC, NADPH-dependent thioredoxin reductase C; ROOH, alkylhydroperoxide with an arbitrary residue $\mathrm{R}$; Trx, thioredoxin.

with only $10 \%$ residual ribulose-1,5-bisphosphate carboxylase/ oxygenase levels suggesting efficient and tight control even under conditions of metabolic imbalance. Chloroplasts contain Fe-dependent and $\mathrm{Cu} / \mathrm{Zn}$-dependent superoxide dismutases (FeSOD1-3, CuZnSOD2 in A. thaliana, Mittler et al., 2004) which catalyze the conversion of superoxide to hydrogen peroxide by the dismutation reaction $2 \mathrm{O}_{2}^{-}+2 \mathrm{H}^{+} \rightarrow \mathrm{H}_{2} \mathrm{O}_{2}+\mathrm{O}_{2}$. Thus the Mehler reaction and PTOX-dependent generation of superoxide and dismutation are considered as major sources of $\mathrm{H}_{2} \mathrm{O}_{2}$ in chloroplasts. Lipid peroxidation occurs if unsaturated fatty acids react with singlet oxygen or superoxide, or by enzymatic catalysis (Farmer and Mueller, 2013). Lipid peroxides initiate radical chain reactions and are degraded to reactive electrophilic species (RES). RES react with macromolecules, alter gene expression and may cause cell damage and thus need to be tightly controlled.

\section{ASCORBATE PEROXIDASES}

Chloroplasts of $A$. thaliana contain two ascorbate peroxidases (APX), a thylakoid-bound tAPX and a soluble sAPX. APXs belong to the group of heme peroxidases that reduce $\mathrm{H}_{2} \mathrm{O}_{2}$ with ascorbate as electron donor (Fig. 1). In the absence of ascorbate nanomolar concentrations of $\mathrm{H}_{2} \mathrm{O}_{2}$ inactivate tAPX. This occurs with a rate constant of $7 \times 10^{5} \mathrm{M}^{-1} \mathrm{~s}^{-1}$ in the presence of $2 \mathrm{nM} \mathrm{H}_{2} \mathrm{O}_{2}$
(Miyake and Asada, 1996). The inactivation occurs if the cosubstrate ascorbate does not convert the reactive intermediate state $\left(\mathrm{Fe}^{\mathrm{IV}}\right)^{\circ+}$ to the safe Fe $\mathrm{Fe}^{\mathrm{III}}$-state of the heme (Fig. 1). Regular stromal ascorbate concentrations are in the range of $10 \mathrm{mM}$, thus the inactivation of TAPX and SAPX can only occur under severe stress conditions such as heavy metal toxicity or severe drought (Liu et al., 2008; Kitajima, 2008). Two reasons are discussed to explain the susceptibility of chloroplast APX to $\mathrm{H}_{2} \mathrm{O}_{2}$-dependent inactivation, the increased turnover rate of plastid APX by adopting more reactive intermediates or the need of inactivation for signaling purposes (Kitajima, 2008). Since electrons are extracted from $\mathrm{H}_{2} \mathrm{O}$ in the water splitting apparatus of photosystem II and finally transferred to $\mathrm{O}_{2}$ in the Mehler reaction, the whole pathway has been termed water-water cycle (Asada, 1999). A conclusive simulation model has been assembled to describe the activity and functional consequences of the ascorbate-dependent water-water cycle in plants (Polle, 2001). This paper provides a survey of reported concentrations of enzymes, activities and substrates of the water water cycle. APX was given with a total concentration of $70 \mu \mathrm{M}, a \mathrm{~K}_{\mathrm{m} \text { (Asc) }}$ of $300 \mu \mathrm{M}$ and the $\mathrm{K}_{\mathrm{m}(\mathrm{H} 2 \mathrm{O} 2)}$ of 30 $\mu \mathrm{M}$ (Nakano and Asada, 1987). The estimated local concentration of tAPX at the thylakoid membrane is $1 \mathrm{mM}$ (Miyake and Asada, 1996). The mathematical model supports the view that the ascorbate and glutathione pools of the chloroplasts can be uncoupled under in vivo conditions (Polle, 2001). 


\section{THIOL PEROXIDASES}

Thiol peroxidases are heme-free enzymes that use a cysteinyl thiolate to attack the peroxide substrates. Any protein that reacts with peroxides via a cysteinyl thiol tentatively might be called thiol peroxidase, e.g. annexins (Dalal et al., 2014) or glutathione-S-transferases (Dixon et al., 2009). However two groups of enzymes, the peroxiredoxins (Prx) and glutathione peroxidases (Gpx), should be considered as thiol peroxidases in sensu stricto due to their high affinity to peroxides. Prx-s were first described as protector proteins and thiol antioxidants in mixed function oxidation assays where DNA and glutamine synthase are degraded in the presence of $\mathrm{Fe}$, thiols and $\mathrm{O}_{2}$ (Kim et al., 1988). Prx-s suppress the degradation process. Higher plants express a set of usually 9 to 10 Prx-s, three to four of which are targeted to plastids (Dietz et al., 2006). The plastid peroxiredoxins belong to the groups of classical 2cysteine peroxiredoxins (2-CysPrx), peroxiredoxin $Q$ (PrxQ) and the type II peroxiredoxin (PrxlIE). Following reaction with peroxide substrate, oxidized 2-CysPrx receives its electrons from NADPH via NADPH-dependent thioredoxin reductase $C$ (NTRC) or from thioredoxins like Trx- $x$ which likely is linked to ferredoxin (Fd) via Fd-dependent thioredoxin reductase (FTR) (Collin et al., 2003; Pulido et al., 2010) (Fig. 1). Work with $A$. thaliana mutants lacking either NTRC or Trx-x suggests that the NTRC-pathway is predominant for regeneration of reduced 2CysPrx (Pulido et al., 2010). If coupled to Trx-dependent regeneration 2-CysPrx equally accepts $\mathrm{H}_{2} \mathrm{O}_{2}$ and small peroxides such as $\mathrm{tBOOH}$, but poorly reacts with lipid peroxides (König et al., 2003). The latter was comfirmed by Award et al. (2015) using dithiothreitol as artificial regenerant. However in this artificial system, $\mathrm{tBOOH}$ reduction only reached about $30 \%$ of the rate of $\mathrm{H}_{2} \mathrm{O}_{2}$ reduction (Award et al., 2015). 2-CysPrx adopts an additional function as chaperone (Jang et al., 2004). Sitedirected mutation of specific amino acids has revealed that chaperone activity of 2-CysPrx does not exclusively depend on the hyperoxidized conformation (König et al., 2013; Lee et al., 2015). PrxllE prefers $\mathrm{H}_{2} \mathrm{O}_{2}>4$-fold over $\mathrm{tBOOH}$ and has negligible rates with cumene hydroperoxide (Treffon and Dietz, un- published). Oxidized PrxllE accepts electrons from glutathione/glutaredoxinC5-system (Couturier et al., 2011). The preferred substrate of PrxQ is $\mathrm{H}_{2} \mathrm{O}_{2}$. Oxidized PrxQ is regenerated by Trx-y (Lamkemeyer et al., 2006). A small set of glutathione peroxidases (Gpx) is targeted to chloroplasts and functions as Trx-dependent peroxide reductases which hardly react with GSH (Navrot et al., 2006; Zhai et al., 2013). Gpx isoforms prefer lipid peroxides as substrates with usually $>10$-fold higher catalytic efficiency towards complex lipid peroxides such as phosphatidylcholine hydroperoxide as compared to $\mathrm{H}_{2} \mathrm{O}_{2}$ (Eshdat et al., 1997; Matamoros et al., 2015).

\section{CONSEQUENCES OF APX, PRX AND GPX DEFICIENCY}

Rice and Arabidopsis plants devoid of chloroplast APX display no altered phenotype under normal growth conditions with constant light, but their development is strongly inhibited in the presence of methylviologen and at low temperature, thus under photoinhibitory conditions (Caverzan et al., 2014; Kangasjärvi et al., 2008). Compensatory upregulation of 2-CysPrx and cytosolic APX may allow for long term high light acclimation of sapx/tapx-plants (Kangasjärvi et al., 2008). Deficiency of 2cysteine peroxiredoxin in antisense $A$. thaliana delays early seedling development with impairment of photosynthesis (Baier and Dietz, 1999; Baier et al., 2000). Growth is inhibited in antisense and T-DNA insertion lines and levels of $\mathrm{H}_{2} \mathrm{O}_{2}$ and carbonylated proteins increase significantly (Awad et al., 2015; Pulido et al., 2010). Photosynthesis of plants lacking 2-CysPrxA and B (2cpa $2 c p b$ ), and in particular of $2 c p a 2 c p b$ tapx-plants which in addition lack thylakoid APX showed decreased quantum yield of photosystem II, and lower $\mathrm{CO}_{2}$ fixation rates. Most importantly, $\mathrm{H}_{2} \mathrm{O}_{2}$-responsive nuclear genes were unchanged in $2 c p a$ $2 c p b$ and only massively accumulated in $2 c p a 2 c p b$ tapx-plants (Awad et al., 2015). The lack of NTRC, the predominant reductant of 2-CysPrx, caused a smaller increase in protein carbonylation of about $36 \%$ of that in 2-cysprx and $64 \%$ increase in $\mathrm{H}_{2} \mathrm{O}_{2}$ (Pulido et al., 2010). Depletion of Gpx1 and Gpx7 from Arabidopsis slightly altered leaf phenotype, compromised acclimation to excess light by increased photoinhibition and $\mathrm{H}_{2} \mathrm{O}_{2}$

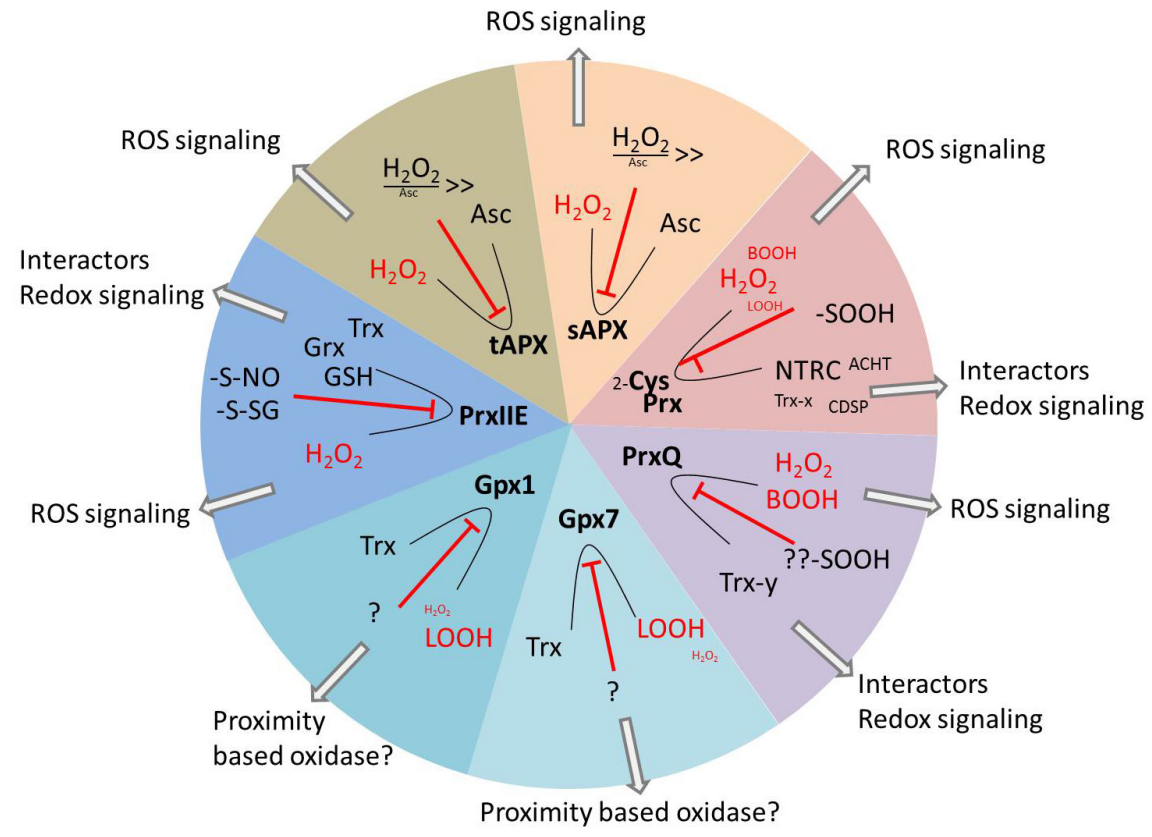

Fig. 2. Overview of peroxidases in chloroplasts. The scheme depicts the name, the regenerators, the substrates and localization of the peroxidases. 2CysPrxA and 2-CysPrxB are considered as highly similar. Abbreviations as in Fig. 1, additional ones: ACHT: chloroplast protein with Trx-domain, CDSP: chloroplastic drought-induced stress protein of $32 \mathrm{kD}$, LOOH: complex lipidhydroperoxides, S-NO: S-nitrosylation, S-SG: S-glutathionylation. The letter size indicates the preference of interaction. 
development, but interestingly has no effect on malondialdehyde (MDA) accumulation (Chang et al., 2009). MDA is a degradation product of lipid peroxidation. Thus other mechanisms appear to counteract the lack of Gpx-dependent lipid peroxide detoxification capacity and possibly are linked to up-regulated levels of ascorbate, glutathione and salicylic acid (Chang et al., 2009).

\section{THE APX, PRX AND GPX NETWORK}

Starting from this biochemical and physiological knowledge one can address the question as to why chloroplasts express so many peroxidases? The answer to this question apart from redundancy must consider eight characteristics and processes, namely (i) suborganellar localization, (ii) substrate preference, (iii) metabolic coupling, (iv) abundance, (v) regulation, (vi) interactions, (vii) signaling functions and (viii) the temporal and spatial requirement for high antioxidant capacity (Fig. 2).

\section{Suborganellar localization}

tAPX and PrxQ are associated with the thylakoids. It still needs to be resolved whether $\operatorname{Pr} X$ associates with the grana stacks from the stromal side, the thylakoid lumen or both (Lamkemeyer et al., 2006; Petersson et al., 2006). 2-CysPrx adopts different conformations in dependence on redox state and experimental evidence suggests that certain 2-CysPrx conformation states associate with thylakoids and photosystem II (König et al., 2003; Muthuramalingam et al., 2009), however these interactions may be short-lived since Cerveau et al. (2016) could not detect major portions of 2-CysPrx at intensively washed thylakoids and also found no correlation with overoxidation state. PrXIIE, SAPX, and Gpx are reported as stromal proteins. It is concluded that part of the peroxidase diversity is due to distinct suborganellar association.

\section{Substrate preference}

Only a limited number of peroxide substrates have been tested with the Prx, Apx and Gpx isoforms. The exclusive specificity of APX for $\mathrm{H}_{2} \mathrm{O}_{2}$, the strong preference of $\mathrm{Gpx}$ for lipid peroxides and the mixed but variable substrate specificity of the Prx-s indicate distinct peroxide detoxification or sensing function.

\section{Metabolic coupling}

The electron donor for APX and the reductants of oxidized Prx and Gpx link peroxide detoxification to different metabolic pathways. Coupling of GSH/ascorbate to APX, NADPH/NTRC and FTR/Trx-y/z/CDSP to 2-CysPrx, PrxQ and also presumably Gpx, and GSH/Grx (as well as presumably FTR/Trx) to PrxIIE diversifies electron drainage and generates robustness in detoxification. Importantly NTRC, but also likely Trx-x, Trx-y and other involved redox transmitters, serve multiple functions in redox regulation. An example is the role of NTRC in regulat- ing photochemical activities of the photosynthetic electron transport chain (Naranjo et al., 2016). Thus diversion of electrons into ROS detoxification via thiol peroxidases may cause spatial oxidation of other target proteins which then alter their function (König et al., 2013).

\section{Protein abundance}

In vivo electron fluxes in the network depend on affinities, rate constants and concentrations of involved players. The abundance of the here discussed participants can be obtained from the At_Chloro database (Ferro et al., 2010) and has recently been assembled (König et al., 2012). The relative scores were described as follows: 2-Cys PRX A (100 relative units, roughly corresponding to concentration in $\mu \mathrm{M})$ : PrxllE (24): PrxQ (8.6):Gpx1 (3.4): sAPX (4.4): tAPX (2.6). Among the redox interactors is Trx-m4 (11 relative units) highly abundant while Trx-x is present with only 0.24 relative units (König et al., 2012). The response of the network to any specific environmental challenge must take into account these distinct abundances. But in addition conditional changes in the relative abundances by quantitative proteomics need to be explored in future work.

\section{Regulation of activity}

It was discussed above that Apx is prone to inactivation in the absence of reduced ascorbate. Likewise, 2-CysPrx is sensitive to hyperoxidation. The peroxidatic Cys which reacts with the peroxide substrate and, thereby is converted to the sulfenic acid derivative within the catalytic cycle, occasionally converts to sulfinic acid. About 250 peroxide reduction reactions have been determined as average catalytic cycles prior to inactivation by hyperoxidation (Liebthal et al., 2016). Thus the rate of peroxide turnover and rereduction of the hyperoxidized form by sulfiredoxin (Liu et al., 2006) determine the active fraction of 2-CysPrx. Interestingly, the fraction of hyperoxidized 2-CysPrx did not strongly change under environmental stress (Cerveau et al., 2016). PrxllE is regulated by Snitrosylation and glutathionylation (Romero-Puertas et al., 2007; Treffon and Dietz, unpublished). Similar data are missing for chloroplast Gpx and PrxQ. But reports from other systems and non-plastidic isoforms suggest that posttranslational modifications also affect the other chloroplast thiol peroxidases (Chae et al., 2012; Seo et al., 2009). These mechanisms likely allow for conditional, spatial and species-specific control of ROS also in chloroplasts.

\section{Interactions with other proteins}

Bacterial, yeast and human Prx and Gpx interact with specific protein partners like receptors and signaling components (Bertoldi, 2016; Flohé, 2015). Plant 2-CysPrx interacts with multiple redox transmitters, but also other proteins such as fructose-1,6bisphosphatase and cyclophilin 20-3 (Caporaletti et al., 2007;

\begin{tabular}{|c|c|c|c|c|c|c|c|c|}
\hline & tAPX & sAPX & 2CPA & 2CPB & PrxQ & PrxllE & Gpx1 & Gpx7 \\
\hline tApx & 1 & 13097 & 241 & 239 & 50 & 1010 & 282 & 2262 \\
\hline sApx & & 1 & 3654 & 5437 & 6281 & 3292 & 20464 & 10083 \\
\hline 2CPA & & & 1 & 32 & 83 & 83 & 1298 & 10087 \\
\hline 2CPB & & & & 1 & 50 & 641 & 1154 & 9875 \\
\hline PrxQ & & & & & 1 & 320 & 1242 & 7881 \\
\hline PrxllE & & & & & & 1 & 3736 & 14568 \\
\hline Gpx1 & & & & & & & 1 & 204 \\
\hline Gpx7 & & & & & & & & 1 \\
\hline
\end{tabular}

Fig. 3. Coexpression among the eight chloroplast peroxidases in A. thaliana. The mutual rank (MR) in the coexpression list was determined using the online tool ATTED-II (http://atted.jp). Each pair of peroxidases was analyzed and MR-values were obtained. The numbers give the rank of the specific MR value compared to that of the bait for all other 22000 gene chip-contained transcripts from 1388 data sets. 
Liebthal et al., 2016; Muthuramalingam et al., 2009). There is a need for the comprehensive search for interaction partners of the various peroxidases, in particular the thiol peroxidases, in order to understand their role in cell signaling. The promiscuity of 2-CysPrx in interacting with thiol proteins like the AtACHTproteins (Dangoor et al., 2009) and chloroplastic droughtinduced stress protein of $32 \mathrm{kD}$ (CDSP32) (Broin et al., 2002) should be considered in both directions; within the catalytic cycle the reduction of oxidized 2-CysPrx enables repeated detoxification of peroxides. In the redox sensory pathway, the oxidation of the thiol protein in dependence on the peroxide concentration may feed information into e.g. the AtACHT pathway and affect cell signaling.

\section{Signaling functions}

Transcript analyses revealed specific alterations of gene expression upon deletion of 2-CysPrx, PrxQ or sAPX/tAPX (Baier and Dietz, 1999; Kangasjärvi et al., 2008; Lamkemeyer et al., 2006). This suggests that each peroxidase is involved in a specific and at least partly unique metabolic and signaling context. Transcriptome profiling of all mutants in a single experiment is needed to ultimately address the distinct and overlapping signaling role. Only a single transcript was significantly up-regulated in the tapx sapx-plants under control conditions (Kangasjärvi et al., 2008). Apparently, the mutant is able to acclimate under constant growth conditions and to compensate for the deletion. Thus transient and fluctuating environmental conditions, respectively, are needed to challenge the mutants and to pinpoint to the molecular and physiological consequences of the specific defects.

\section{Conditional requirement for specific antioxidant capacity}

Both tapx sapx- and 2cysprx-plants revealed chlorotic phenotype and growth inhibition during early seedling development (Baier et al., 1999; Kangasjärvi et al., 2008). Chloroplast APX activity dropped to very low levels at 2 to $3 \mathrm{~d}$ after radicle emergence (Pena-Ahumada et al., 2006). The authors concluded that the ascorbate-dependent water-water cycle only develops following a metabolic switch at 3 day after radicle emergence whereas Prx-s might be important during very early development (Pena-Ahumada et al., 2006). Similar versus dissimilar regulation can be deduced from transcriptome data sets by applying coexpression analysis tools (e.g. at ATTED-II, Obayashi et al., 2009). Figure 3 depicts the Mutual Rank (MR) among the pairwise comparison for coexpression following sorting from the strongest to the weakest co-expression regulation. Low numbers indicate high degree of coexpression. It can be seen that there is significant coregulation between 2-CysPrxA, 2CysPrxB and PrxQ. Some coexpression is observed between Prx and tApx, but most other pairs of peroxidases reveal negligible coexpression in transcript regulation. The result shows that the peroxidases are specifically regulated in dependence on environmental and developmental cues. In summary the available evidence supports the eight hypotheses explaining why plants rely on multiple peroxidase systems in the photosynthesizing chloroplast.

\section{ACKNOWLEDGMENTS}

The own work cited in this review originates from projects funded by the Deutsche Forschungsgemeinschaft (DI346, FOR804, SPP1710). Support by Dr. Thorsten Seidel in coexpression analysis is acknowledged.

\section{REFERENCES}

Allahverdiyeva, Y., Suorsa, M., Tikkanen, M., and Aro, E.M. (2015).
Photoprotection of photosystems in fluctuating light intensities. J. Exp. Bot. 66, 2427-2436.

Asada, K. (1999). The water-water cycle in chloroplasts: Scavenging of active oxygens and dissipation of excess photons. Annu. Rev. Plant Physiol. Plant Mol. Biol. 50, 601-639.

Awad, J., Stotz, H.U., Fekete, A., Krischke, M., Engert, C., Havaux, M., Berger, S., and Mueller, M.J. (2015). 2-cysteine peroxiredoxins and thylakoid ascorbate peroxidase create a water-water cycle that is essential to protect the photosynthetic apparatus under high light stress conditions. Plant Physiol. 167, 1592-1603.

Badger, M.R., von Caemmerer, S., Ruuska, S., and Nakano, H. (2000). Electron flow to oxygen in higher plants and algae: rates and control of direct photoreduction (Mehler reaction) and Rubisco oxygenase. Philos. Trans. R. Soc. B: Biol. Sci. 355, 1433-1446.

Baier, M., and Dietz, K.J. (1999). Protective function of chloroplast 2-Cys peroxiredoxin in photosynthesis: Evidence from transgenic Arabidopsis thaliana. Plant Physiol. 119, 1407-1414.

Baier, M., Noctor, G., Foyer, C.H., and Dietz, K.J. (2000). Antisense suppression of 2-Cys peroxiredoxin in Arabidopsis thaliana specifically enhances the activities and expression of enzymes associated with ascorbate metabolism, but not glutathione metabolism. Plant Physiol. 124, 823-832.

Bertoldi, M. (2016). Human peroxiredoxins 1 and 2 and their interacting protein partners; through structure toward functions of biological complexes. Protein Pept. Lett. [in press]

Broin, M., Cuiné, S., Eymery, F., and Rey, P. (2002). The plastidic 2cysteine peroxiredoxin is a target for a thioredoxin involved in the protection of the photosynthetic apparatus against oxidative damage. Plant Cell 14, 1417-1432.

Caporaletti, D., D'Alessio, A.C., Rodriguez-Suarez, R.J., Senn, A.M., Duek, P.D., and Wolosiuk, R.A. (2007). Non-reductive modulation of chloroplast fructose-1,6-bisphosphatase by 2-Cys peroxiredoxin. Biochem. Biophys. Res. Commun. 355, 722-727.

Caverzan, A., Bonifacio, A., Carvalho, F.E., Andrade, C.M., Passaia, G., Schünemann, M., Maraschin Fdos, S., Martins, M.O., Teixeira, F.K., Rauber, R., et al. (2014). The knockdown of chloroplastic ascorbate peroxidases reveals its regulatory role in the photosynthesis and protection under photo-oxidative stress in rice. Plant Sci. 214, 74-87.

Cerveau, D., Ouahrani, D., Marok, M.A., Blanchard, L., and Rey, P. (2016). Physiological relevance of plant 2-Cys peroxiredoxin overoxidation level and oligomerization status. Plant Cell Environ. [in press]

Chae, H.Z., Oubrahim, H., Park, J.W., Rhee, S.G., and Chock, P.B. (2012). Protein glutathionylation in the regulation of peroxiredoxins: a family of thiol-specific peroxidases that function as antioxidants, molecular chaperones, and signal modulators. Antioxid. Redox. Signal. 16, 506-523.

Chang, C.C., Slesak, I., Jordá, L., Sotnikov, A., Melzer, M., Miszalski, Z., Mullineaux, P.M., Parker, J.E., Karpinska, B., and Karpinski, S. (2009). Arabidopsis chloroplastic glutathione peroxidases play a role in cross talk between photooxidative stress and immune responses. Plant Physiol. 150, 670-683.

Collin, V., Issakidis-Bourguet, E., Marchand, C., Hirasawa, M., Lancelin, J.M., Knaff, D.B., and Miginiac-Maslow, M. (2003). The Arabidopsis plastidial thioredoxins: new functions and new insights into specificity. J. Biol. Chem. 278, 23747-23752.

Couturier, J., Ströher, E., Albetel, A.N., Roret, T., Muthuramalingam, M., Tarrago, L., Seidel, T., Tsan, P., Jacquot, J.P., Johnson, M.K. et al. (2011). Arabidopsis chloroplastic glutaredoxin C5 as a model to explore molecular determinants for iron-sulfur cluster binding into glutaredoxins. J. Biol. Chem. 286, 27515-27527.

Dalal, A., Vishwakarma, A., Singh, N.K., Gudla, T., Bhattacharyya, M.K., Padmasree, K., Viehhauser, A., Dietz, K.J., and Kirti, P.B. (2014). Attenuation of hydrogen peroxide-mediated oxidative stress by Brassica juncea annexin-3 counteracts thiol-specific antioxidant (TSA1) deficiency in Saccharomyces cerevisiae. FEBS Lett. 588, 584-593.

Dangoor, I., Peled-Zehavi, H., Levitan, A., Pasand, O., and Danon, A. (2009). A small family of chloroplast atypical thioredoxins. Plant Physiol. 149, 1240-1250.

Dietz, K.J., Jacob, S., Oelze, M.L., Laxa, M., Tognetti, V., de Miranda, S.M., Baier, M., and Finkemeier, I. (2006). The function of peroxiredoxins in plant organelle redox metabolism. J. Exp. Bot. 57, 1697-1709.

Dietz, K.J., and Hell, R. (2015). Thiol switches in redox regulation of 
chloroplasts: balancing redox state, metabolism and oxidative stress. Biol Chem. 396, 483-494.

Dixon, D.P., Hawkins, T., Hussey, P.J., and Edwards, R. (2009). Enzyme activities and subcellular localization of members of the Arabidopsis glutathione transferase superfamily. J. Exp. Bot. 60, 1207-1218.

Eshdat, Y., Holland, D., Faltin, Z., and BenHayyim, G. (1997). Plant glutathione peroxidases. Physiol. Plant 100, 234-240.

Farmer, E.E., and Mueller, M.J. (2013). ROS-mediated lipid peroxidation and RES-activated signaling. Annu. Rev. Plant Biol. 64, 429-450.

Ferro, M., Brugiere, S., Salvi, D., Seigneurin-Berny, D., Court, M., Moyet, L., Ramus, C., Miras, S., Mellal, M., Le Gall, S., et al. (2010). AT CHLORO, a comprehensive chloroplast proteome database with subplastidial localization and curated information on envelope proteins. Mol. Cell Proteomics 9, 1063-1084.

Flohé, L. (2015). The impact of thiol peroxidases on redox regulation. Free Radic. Res. 14, 1-17.

Heyno, E., Gross, C.M., Laureau, C., Culcasi, M., Pietri, S., and Krieger-Liszkay, A. (2009). Plastid alternative oxidase (PTOX) promotes oxidative stress when overexpressed in tobacco. J. Biol. Chem. 284, 31174-31180.

Jang, H.H., Lee, K.O., Chi, Y.H., Jung, B.G., Park, S.K., Park, J.H., Lee, J.R., Lee, S.S., Moon, J.C., Yun, J.W., et al. (2004). Two enzymes in one; two yeast peroxiredoxins display oxidative stress-dependent switching from a peroxidase to a molecular chaperone function. Cell 117, 625-635.

Kangasjärvi, S., Lepistö, A., Hännikäinen, K., Piippo, M., Luomala, E.M., Aro, E.M., and Rintamäki, E. (2008). Diverse roles for chloroplast stromal and thylakoid-bound ascorbate peroxidases in plant stress responses. Biochem. J. 412, 275-285.

Kim, K., Kim, I.H., Lee, K.Y., Rhee, S.G., and Stadtman, E.R. (1988) The isolation and purification of a specific "protector" protein which inhibits enzyme inactivation by a thiol/Fe(III)/O2 mixedfunction oxidation system. J. Biol. Chem. 263, 4704-4711.

Kitajima, S. (2008). Hydrogen peroxide-mediated inactivation of two chloroplastic peroxidases, ascorbate peroxidase and 2-cys peroxiredoxin. Photochem. Photobiol. 84, 1404-1409.

König, J., Lotte, K., Plessow, R., Brockhinke, A., Baier, M., and Dietz, K.J. (2003). Reaction mechanism of the 2-Cys peroxiredoxin: Role of the C-terminus and the quarternary structure. J. Biol. Chem. 278, 24409-24420.

König, J., Muthuramalingam, M., and Dietz, K.J. (2012). Mechanisms and dynamics in the thiol/disulfide redox regulatory network: transmitters, sensors and targets. Curr. Opin. Plant Biol. $15,261-268$

König, J., Galliardt, H., Jütte, P., Schäper, S., Dittmann, L., and Dietz, K.J. (2013). The conformational bases for the two functionalities of 2-Cys peroxiredoxins as peroxidase and chaperone. $\mathrm{J}$. Exp. Bot. 64, 3483-3497.

Laisk, A., Eichelmann, H., Oja, V., Rasulov, B. ,and Rämma, H. (2006). Photosystem II cycle and alternative electron flow in leaves. Plant Cell Physiol. 47, 972-983.

Lamkemeyer, P., Laxa, M., Collin, V., Li, W., Finkemeier, I., Schöttler, M.A., Holtkamp, V., Tognetti, V.B., Issakidis-Bourguet, E., Kandlbinder, A., et al. (2006). Peroxiredoxin Q of Arabidopsis thaliana is attached to the thylakoids and functions in context of photosynthesis. Plant J. 45, 968-981.

Lee, E.M., Lee, S.S., Tripathi, B.N., Jung, H.S., Cao, G.P., Lee, Y., Singh, S., Hong, S.H., Lee, K.W., Lee, S.Y., et al. (2015). Sitedirected mutagenesis substituting cysteine for serine in 2-Cys peroxiredoxin (2-Cys Prx A) of Arabidopsis thaliana effectively improves its peroxidase and chaperone functions. Ann. Bot. 116, 713-725.

Liebthal, M., Strüve, M., Li, X., Hertle, Y., Maynard, D., Hellweg, T., Viehhauser, A., and Dietz, K.J. (2016). Redox-dependent conformational dynamics of decameric 2-cysteine peroxiredoxin and its interaction with cyclophilin Cyp20-3. Plant Cell Physiol. resubmitted, provisional acceptance.

Liu, X.P., Liu, X.Y., Zhang, J., Xia, Z.L., Liu, X., Qin, H.J., and Wang, D.W. (2006). Molecular and functional characterization of sulfiredoxin homologs from higher plants. Cell Res. 16, 287-296.

Liu, K.L., Shen, L., Wang, J.Q., and Sheng, J.P. (2008). Rapid inactivation of chloroplastic ascorbate peroxidase is responsible for oxidative modification to Rubisco in tomato (Lycopersicon escu- lentum) under cadmium stress. J. Integr. Plant Biol. 50, 415-426. Matamoros, M.A., Saiz, A., Peñuelas, M., Bustos-Sanmamed, P., Mulet, J.M., Barja, M.V., Rouhier, N., Moore, M., James, E.K. Dietz, K.J., et al. (2015). Function of glutathione peroxidases in legume root nodules. J. Exp. Bot. 66, 2979-2990.

Mittler, R., Vanderauwera, S., Gollery, M., and Van Breusegem, F. (2004). Reactive oxygen gene network of plants. Trends Plant Sci. 9, 490-498.

Miyake, C., and Asada, K. (1996) Inactivation mechanism of ascorbate peroxidase at low concentrations of ascorbate; hydrogen peroxide decomposes compound I of ascorbate peroxidase. Plant Cell Physiol. 37, 423-430.

Muthuramalingam, M., Seidel, T., Laxa, M., Nunes de Miranda, S., Gärtner, F., Ströher, E., Kandlbinder, A., and Dietz, K.J. (2009) Multiple redox and non-redox interactions define 2-Cys peroxiredoxin as a regulatory hub in the chloroplast. Mol. Plant 2, 1273-1288.

Nakano, Y., and Asada, K. (1987). Purification of ascorbate peroxidase in spinach chloroplasts; its inactivation in ascorbatedepleted medium and reactivation by monodehydroascorbate radical. Plant Cell Physiol. 28, 131-140.

Navrot, N., Collin, V., Gualberto, J., Gelhaye, E., Hirasawa, M., Rey, P., Knaff, D.B., Issakidis, E., Jacquot, J.P., and Rouhier, N (2006). Plant glutathione peroxidases are functional peroxiredoxins distributed in several subcellular compartments and regulated during biotic and abiotic stresses. Plant Physiol. 142, 13641379.

Naranjo, B., Mignée, C., Krieger-Liszkay, A., Hornero-Méndez, D., Gallardo-Guerrero, L., Cejudo, F.J., and Lindahl, M. (2016). The chloroplast NADPH thioredoxin reductase C, NTRC, controls non-photochemical quenching of light energy and photosynthetic electron transport in Arabidopsis. Plant Cell Environ. [in press].

Obayashi, T., Hayashi, S., Saeki, M., Ohta, H., and Kinoshita, K. (2009). ATTED-II provides coexpressed gene networks for Arabidopsis. Nucleic Acids Res. 37, D987-91.

Pena-Ahumada, A., Kahmann, U., Dietz, K.J., and Baier, M. (2006). Antioxidant defence in seedling development of Arabidopsis thaliana. Photosynthesis Res. 89, 99-112.

Petersson, U.A., Kieselbach, T., García-Cerdán, J.G., and Schröder, W.P. (2006). The Prx Q protein of Arabidopsis thaliana is a member of the luminal chloroplast proteome. FEBS Lett. 580, 6055-6061

Polle, A. (2001). Dissecting the superoxide dismutase-ascorbateglutathione-pathway in chloroplasts by metabolic modeling. Computer simulations as a step towards flux analysis. Plant Physiol. 126, 445-462

Pulido, P., Spínola, M.C., Kirchsteiger, K., Guinea, M., Pascual, M.B., Sahrawy, M., Sandalio, L.M., Diet,z .K.J, González, M., and Cejudo, F.J. (2010). Functional analysis of the pathways for 2-Cys peroxiredoxin reduction in Arabidopsis thaliana chloroplasts. J. Exp. Bot. 61, 4043-4054.

Romero-Puertas, M.C., Laxa, M., Mattè, A., Zaninotto, F., Finkemeier, I., Jones, A.M., Perazzolli, M., Vandelle, E., Dietz, K.J. and Delledonne, M. (2007). S-nitrosylation of peroxiredoxin II E promotes peroxynitrite-mediated tyrosine nitration. Plant Cell 19, 4120-4130.

Ruuska, S.A., Badger, M.R., Andrews, T.J., and von Caemmerer, S. (2000). Photosynthetic electron sinks in transgenic tobacco with reduced amounts of Rubisco: little evidence for significant Mehler reaction. J. Exp. Bot. 51, 357-368.

Seo, J.H., Lim, J.C., Lee, D.Y., Kim, K.S., Piszczek, G., Nam, H.W., Kim, Y.S., Ahn, T., Yun, C.H., Kim, K., et al. (2009). Novel protective mechanism against irreversible hyperoxidation of peroxiredoxin: N-alpha-terminal acetylation of human peroxiredoxin II. J. Biol. Chem. 284, 13455-13465.

Shirao, M., Kuroki, S., Kaneko, K., Kinjo, Y., Tsuyama, M., Förster, B., Takahashi, S., and Badger, M.R. (2013). Gymnosperms have increased capacity for electron leakage to oxygen (Mehler and PTOX reactions) in photosynthesis compared with angiosperms. Plant Cell Physiol. 54, 1152-1163.

Zhai, C.Z., Zhao, L., Yin, L.J., Chen, M., Wang, Q.Y., Li, L.C., Xu, Z.S., and You-Zhi Ma, Y.Z. (2013). Two wheat glutathione peroxidase genes whose products are located in chloroplasts improve salt and $\mathrm{H}_{2} \mathrm{O}_{2}$ tolerances in Arabidopsis. PLoS One 8, e73989. 